\title{
AAPS Workshop: accelerating pharmaceutical development through predictive stability approaches, April 4-5, 2016
}

\author{
A. L. Freed ${ }^{1}$, S. T. Colgan ${ }^{1}$, J. D. Kochling ${ }^{2}$ and M. S. Alasandro ${ }^{3 *}$
}

\begin{abstract}
There has been significant growth in the use of modeling tools to accelerate development and enhance pharmaceutical quality. Among these are empirical and semi-empirical modeling of accelerated stability studies which can be used to predict product shelf-life (Waterman, Pharm Res 24:780-790, 2007; (Wu et al., AAPS Pharm Sci Tech,16:986-991, 2016); (Lavrich, Rapid Development of Robust Stability Models Using Semi-Empirical Design Space, AAPS Webinar, 2016)). These approaches coupled with Lean Stability and Quality by Design (QbD) Analytical Method Development have been discussed at a recent AAPS Workshop (AAPS Workshop, Accelerating Pharmaceutical Development through Predictive Stability Approaches, 2016) with subsequent discussions at a Face-to-Face Focus Group meeting (AAPS, Joint Face-toFace meeting summary, 6-April, part1, 2017; AAPS, Joint Face-to-Face meeting summary, 6-April, Part II, 2017; Huynh-Ba, et al., Meeting Report, Analytical Approaches to Ensure Product Quality - AAPS Joint Face-to-Face Meeting of the Stability, the Pharmaceutical Impurities, and the CMC Statistics Focus Group, 2017) and the AAPS Annual meeting (AAPS Annual Meeting Sunrise Session, Accelerating Pharmaceutical Development through Predictive Stability Approaches, AAPS Annual Meeting, 2016). A summary/overview and the outcome from the workshop are captured in this publication. The first part focuses on use of Lean Stability Strategies to expedite the development of new chemical entities in early phase development, and to facilitate process, formulation for various dosage forms (solid, liquid, etc.) and product changes throughout development and post-approval. Also, presented, are the global regulatory agencies feedback, challenges and future direction. For Lean Stability and predictive stability approaches to be successful, the methods used must be accurate, precise and consistent across stability time points, analysts, instruments and testing sites, so the data and predictions are meaningful. For both small and large molecules, the use of QbD for analytical method development aids in achieving this goal; and, the second part of this publication discusses such approaches. These approaches are essential to build an overall optimized stability strategy to meet today's challenges to advance new medicines and enhance quality.
\end{abstract}

Keywords: Stability modeling, Analytical QbD, Dissolution modeling

\section{Background}

The workshop on "Accelerating Pharmaceutical Development through Predictive Stability Approaches" was held April 4-5, 2016 at the USP Conference Center in Rockville, MD (AAPS Workshop, 2016). The meeting had over 88 attendees with subject matter experts from industry, USP and the FDA discussing the latest

\footnotetext{
* Correspondence: malasandro@orexigen.com

3Orexigen Therapeutics, Inc, La Jolla, CA 92037, USA

Full list of author information is available at the end of the article
}

innovations in predictive stability modeling, lean stability strategies and applications of $\mathrm{QbD}$ analytical development for large and small molecules. This meeting was immediately followed by a Face-to-Face meeting at the Medimmune facility in Gathersburg, MD on April 6, 2016 (AAPS, 2017a; AAPS, 2017b; Huynh-Ba et al., 2017). The outcomes from these meetings and an update on predictive stability approaches was presented at an Annual AAPS meeting Sunrise Session in Denver, CO on Oct 16, 2016 (AAPS Annual Meeting Sunrise Session, 
2016) to over 200 attendees. This paper presents a summary of the workshop's discussion on lean stability strategies and the use of $\mathrm{QbD}$ for analytical method development. Recent publications have discussed advances in predictive dissolution, stability and analytical method modeling (Li et al., 2016; Lavrich, 2016; Wu et al, 2016; Alasandro \& Little, 2014; Alasandro et al., 2013; Alasandro and Little, 2016).

\section{Part I}

Lean stability: integrating modeling tools and regulatory reception as prepared by A. L. Freed and S. T. Colgan, Pfizer WRD, Groton CT

\section{Introduction}

"Lean" in a general sense, seeks to maximize customer value while minimizing waste. A popular misconception is that lean principles can only be applied to manufacturing. This is not a true statement, as lean concepts can be applied to every business and process. Lean is not a tactic or cost reduction program, but a way of thinking and acting. Lean stability, while currently in scope of many regulatory guidances represents a new and evolving concept. Lean strategies can facilitate the development and approval of new and improved medicines by emphasizing the key elements that contribute to quality, safety and efficacy while deemphasizing elements that do not.

A lean stability strategy is science- and risk-based, providing focus on meaningful attributes and time points. A lean strategy could include adjustments to stability protocols or strategies to improve efficiency and expedite stability results. Lean stability strategies could result in less frequent and/or delayed pull points, fewer stability conditions, and leaner analytical test profiles that focus on the individual product's stability related quality attributes (SRQA): and, ideally include only the shelf-life limiting attributes (SLLA) (Table 1).

Lean stability concepts were first included in guidance with the 2002 publication of the International Conference on Harmonization's Q1D guidance on bracketing and matrixing (ICH Q1D, 2002). ICH Q1A(R2), published in 2003 also allows lean strategies as "Alternative approaches can be used when there are scientifically justifiable reasons" (ICH Q1A(R2), 2003). In spite of the flexibility allowed by guidance, bracketing, matrixing, and other lean strategies remain under-utilized. Interestingly, the increasing acceptance of the concept of realtime release testing has helped to spur the growth of lean stability testing. In this scenario, when pharmaceutical products finish production, they can be released without additional testing because quality has been built into the product. Leveraging the momentum supplied by real time release testing, industry and regulators should work together to collectively know so much about the process, product and its stability that when the product is produced, the sponsor can guarantee that the product will be within specification at the end of shelf-life without needing to do any testing to prove it and the regulators are comfortable with this position. While it is unlikely that we will achieve this utopian vision of stability testing in the near future, the vision is directionally correct and in line with the FDA's Pharmaceutical Quality for the twenty-first Century Initiative which advocates a robust quality system and may serve to lower the need for regulatory oversight, allowing for more efficient, focused inspections and less review oversight (FDA, 2004).

Implementation of modeling tools can be key to understanding the drug substance (DS) and/or drug

Table 1 List of Acronyms

\begin{tabular}{|c|c|}
\hline ALCM & Analytical Life Cycle Management \\
\hline AQbD & Analytical Quality by Design \\
\hline ASAP & Accelerated Stability Assessment Program \\
\hline ATP & Analytical Target Profile \\
\hline CBE & Change Being Effective \\
\hline CE-SDS & Capillary Electrophoresis - Sodium dodecyl sulfate \\
\hline CMA & Critical Method Attribute \\
\hline CQA & Critical Quality Attribure \\
\hline CTA & Clinical Trial Application \\
\hline DP & Drug Product \\
\hline DS & Drug Substance \\
\hline EM & Emerging Market \\
\hline EU & European Union \\
\hline IMPD & Investigational Medicinal Product Dossier \\
\hline IQ & Inovation and Quality \\
\hline IR & Immediate Release \\
\hline KMP & Key Method Parameter \\
\hline MA & Marketing Application \\
\hline MR & Modified Release \\
\hline NCE & new chemical entity \\
\hline PA & Post Approval \\
\hline PAC & Post Approval Change \\
\hline PAS & prior approval supplement \\
\hline PCV & Polychlorovinyl \\
\hline QbD & Quality by Design \\
\hline SLLA & shelf-life limiting attributes \\
\hline SRA & Stability Risk Assessment \\
\hline SRQA & stability related quality attributes \\
\hline UHPLC & Ultra High Pressure Liquid Chromatography \\
\hline WRD & Worldwide Research and Development \\
\hline
\end{tabular}


product (DP) stability. A discussion of these and the resulting output can be included in regulatory dossiers throughout a substance's/product's lifecycle for justifying the implementation of lean stability strategies. The primary focus and application experience has been with small molecules, i.e. new chemical entities (NCEs). However, stability modeling tools and approaches may be applied to all types of products, including biologics, thereby allowing the removal of one or more stability tests or time points if scientifically justified.

\section{Integrating stability modeling tools into a lean stability design}

Adoption of lean strategies during product development and in regulatory filings can be facilitated by leveraging stability modeling tools, such as shown in the four case studies below.

\section{Case study 1: using ASAP to determine the shelf-life limiting attribute}

Previously it has been reported that ASAP (Waterman et al., 2007) can be used to show that the degradant for a product represents the shelf-life limiting attribute while the assay does not (Colgan et al., 2015; Colgan et al., 2014; Langer et al., 2016). In one case (Colgan et al., 2014), storage at $40{ }^{\circ} \mathrm{C} / 75 \% \mathrm{RH}$ in PVC/Aclar 2000 blisters would result in significant degradation within 6 months and that this packaging option would result in an unacceptably short shelf life in Zone $4 \mathrm{~b}$. In contrast, after storage for 36 months $30{ }^{\circ} \mathrm{C} / 75 \% \mathrm{RH}$ the assay value would be well within the acceptance criteria of $90-100 \%$ of label claim. Under the same conditions, the degradant is at higher risk of breaching its proposed acceptance criteria of $1.0 \%$ at 36 months. For this product, the degradant represents the shelf-life limiting attribute while the assay does not and these data would help support a post-approval proposal to monitor only the degradant.

\section{Cast study 2: leveraging statistics to justify removing DP Assay as an attribute tested in annual batches}

In 2011, the initial NDA for a stable compound was submitted and proposed lean post- approval stability strategies for both drug substance and drug product. At approval, the FDA agreed to remove water content, disintegration, tablet hardness from the DP protocol, but they were not comfortable removing assay or microbial limits from the DP protocols (Langer et al., 2016).

With full shelf life data on all registration and commercial validation lots in-hand, Pfizer will ask the FDA to reconsider a lean stability proposal for future annual commitment lots and variations (Langer et al., 2016). For the DP assay, batch data were pooled by tablet strength, shape, and packaging configuration and statistically evaluated to predict how long assay would remain within specification.

All assay values were well separated from the lower acceptance criteria of $90 \%$ label claim and when all batches are considered, the statistical projected shelf-life based on assay will range from a worst case of 19 years to as long as 50 years. Since the approved shelf-life of this product is 3 years, the likelihood of any batch failing assay on stability is remote and Pfizer will use these data to help justify removal of assay as an attribute tested on stability.

Case study 3: predicting degradation pathways using zeneth Zeneth (Zeneth by Lhasa Limited, 2017) is an expert, rules-based computer system to predict the forced degradation pathways of organic compounds in solution. A wide variety of stress conditions can be probed including acid, base, peroxide, radical initiator, oxygen, metals, light and these will predict degradation products from DS only or DS plus excipients and/or excipient impurities and/or solvents and/or counter-ions. Each degradation pathway is assigned relative likelihood of happening. The output can help identify potential liabilities of DS \& products regarding degradation and can enable excipient selection and formulation development.

\section{Case study 4: dissolution stability modeling}

Currently there are no general models that can accurately predict how dissolution rate will change over time. Because of this, it is difficult to classify dissolution as an attribute that will not limit shelf life. Dissolution rate testing (which is the most expensive test to run) appears on virtually every solid dosage form drug product stability protocol unless disintegration can be justified as a surrogate. Preliminary data has been presented (Colgan, 2016) that attempted to answer the question of "Can short-term challenge conditions be developed that will be able to accurately predict a dissolution change with stability?"

Figure 1 illustrates dissolution failures as a function of time at $80 \% \mathrm{RH}$. Tablets were challenged at temperatures changing from $30^{\circ} \mathrm{C}$ to $80^{\circ} \mathrm{C}$ and relative humidities ranging from 65 to $90 \% \mathrm{RH}$. These data were fitted using an ASAPprime modified Arrhenius equation and were used to predict when dissolution would fail as a function of packaging and storage condition. For this specific product, these preliminary data indicate that dissolution is not the attribute that will limit shelf life (a degradant is). This information could be used as part of a justification to remove dissolution rate testing from stability protocols.

\section{Global regulatory reception of lean stability strategies - successes and challenges}

The goal is to use science- and risk-based approaches to meet regulatory expectations for stability requirements 


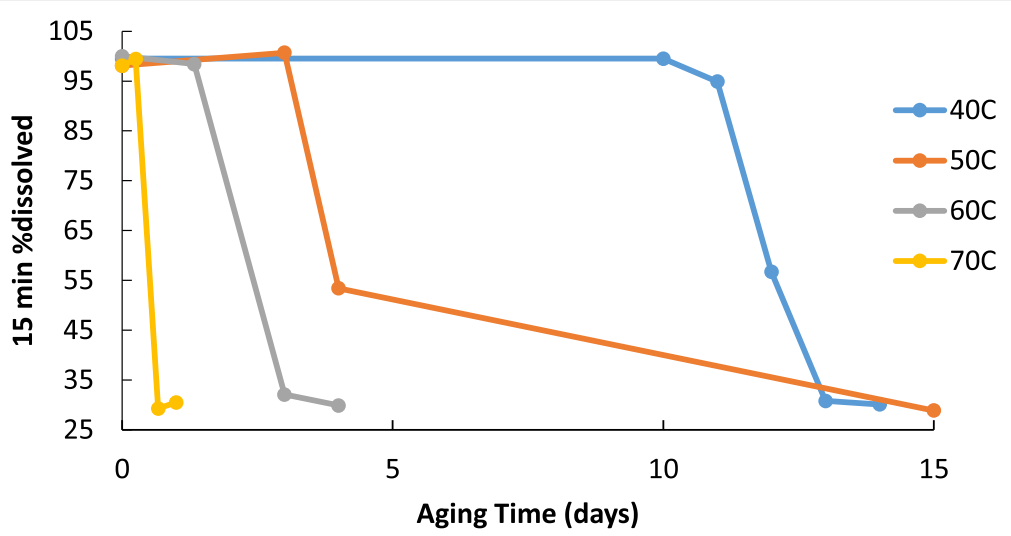

Fig. 1 Dissolution behavior of an immediate release tablets as a function of time, for several temperatures (at 80\%RH). (permitted to reprint)

and to enable a more efficient and expeditious availability of product to patients. The use of such strategies provides benefit to industry, regulatory agencies and patients (Freed, 2016).

\section{Potential lean stability strategies in clinical development}

Clinical regulatory guidances are generally not prescriptive and global expectations vary (CHMP/QWP/185401/ 2004, 2006; FDA Guidance for Industry, 1995; FDA Guidance for Industry, 2003; Health Canada Quality, 2013). Expectations in some cases depend on the phase of development; whereas, others may expect alignment with ICH Q1A (ICH Q1D, 2002) for registration. Examples of potential lean stability strategies and whether related information is included in the clinical trial application (CTA; i.e. IND, IMPD) are included in Table 2 below (Freed, 2016; Freed et al., 2014; Timpano et al., 2017).

These strategies have been prosecuted for ten plus years; and, have been included in clinical filings in many regionals (USA, EU, Canada, Emerging Markets). Overall, they have been successful in most cases. In instances where queries or comments were received from Health Authorities, resolution was reached by providing additional information (Freed, 2016; Freed et al., 2014).
Case study 1 ASAP was used to assess whether a change in bond formation chemistry impacted the stability of a drug substance. This information was included in CTAs to solely support maintaining the 60 month retest period for a global phase 3 study filed in approximately 25 countries (Freed, 2016; Timpano et al., 2017). The only query came from the China Health Authority, whom did not question the review period but did request that the material using the new chemistry be placed on confirmatory stability. This approach has been used globally for other products at various stages of clinical development and overall has been successful in most cases.

Case study 2 The use of ASAP as sole support to assign an initial 12 month use period for DP in global CTAs to support a phase 2 clinical study. In this case, the Serbia Health Authority requested additional stability data and that the testing and extension protocol align with $\mathrm{ICH}$ Q1 guidelines, but the use period was not questioned.

Case study 3 ASAP was employed to demonstrate the change in tablet film-coat color had no impact on the stability of a DP for an initial phase 3 study obviating the need for a stability study. As such, the stability of a

Table 2 Examples of Clinical Lean Stability Strategies

\begin{tabular}{|c|c|c|}
\hline No. & Strategy & Comments \\
\hline 1 & $\begin{array}{l}\text { Apply DS stability data (solely) to simplified/DS-based dosage forms, e.g. DS in a } \\
\text { bottle or capsule }\end{array}$ & $\begin{array}{l}\text { Justification included in the CTA (also reference } \\
\text { section S.7 in P.8) }\end{array}$ \\
\hline 2 & Assign initial DS review period of $18 \mathrm{M}$ based on one week $70^{\circ} \mathrm{C} / 75 \% \mathrm{RH}$ data & Commitment for 'formal' program included in CTA \\
\hline 3 & $\begin{array}{l}\text { Use ASAP to determine packaging, long-term storage conditions, to set the } \\
\text { initial use period (e.g. } 12 \text { month) }\end{array}$ & $\begin{array}{l}\text { Data, conclusions and commitment for 'formal' } \\
\text { program included in CTA }\end{array}$ \\
\hline 4 & $\begin{array}{l}\text { Use ASAP to assist in assessing whether a change in DS or DP process, composition } \\
\text { or packaging affects stability, and thus need for additional stability program }\end{array}$ & Inclusion in CTA is case dependent \\
\hline 5 & Reduce testing protocol (e.g. time points, tests, conditions) based on supportive data & $\begin{array}{l}\text { Inclusion of explicit justification in the CTA is } \\
\text { case dependent }\end{array}$ \\
\hline 6 & Eliminate DS assay testing if it is the same lot as the reference standard & Justification included in the CTA \\
\hline
\end{tabular}


development lot of blue film-coated tablets was used to set the use period for the white film-coated tablets to be used in the clinical study. A commitment to test the clinical tablets was included with reduced conditions and time points for the long-term condition of $30{ }^{\circ} \mathrm{C} / 75 \% \mathrm{RH}$. There were no comments or queries for this global filing, with submissions in approximately 24 countries. This approach was further tested in filings for a subsequent phase 3 study where the tablets used a different film-coat. In this case, the ASAP information was not provided and the development lots were continued to be used to set the use period.

Case study 4 Assay is excluded when the working standard is the same batch as that which is on stability, i.e. there is only one batch manufactured to date. The justification for not including assay in the stability program was included in the CTAs to support the phase 2 study and no comments/queries were received. This approach has been used globally and has been successful in most cases, except for Canada. For one product, Belgium Health Authority asked this test to be included in future submission for a product. The Czech Republic Health Authority was provided information using the mass balance approach (assay $=100 \%$-percent total degradants) and required a commitment to monitor the test on a future lot.

\section{Potential lean stability strategies for post-approval commitments}

Registration guidances generally include prescriptive stability expectations/requirements. Several (e.g. country and/or regional) also do include use of alternative approaches when scientifically justified, e.g. language is present in $\mathrm{ICH}$ $\mathrm{Q} 1 \mathrm{~A}(\mathrm{R} 2)$ (ICH Q1A(R2), 2003), the WHO (WHO guideline, 2009) and Emerging markets (EMs). However, there are often numerous country-specific regulations that are contradictory to the applicable regional guidances. In the EMs, there often times are specific requirements beyond those in the major markets. Further, the expectations can be very "dynamic" and each reviewer's opinion may vary. But, there is an opportunity here, as often time regulators are open and inviting for discussions and show interest in science- and risk-based approaches.

Table 3 illustrates several potential strategies for inclusion in the initial and post-approval (PA) registration dossiers globally (Colgan et al., 2014; Freed, 2016; Timpano et al., 2017). In all cases, the justification is included in the submission. These proposals should be the easiest to justify due to the vast amount of product knowledge and stability data available to leverage. They can be used and filed globally for drug substances and drug products (e.g. standard, conventional immediate release to complex combination modified release products).
In registration and post-approval dossiers, success has been variable and inconsistencies between agencies and in some cases within the same agency (on different products) have been observed. Lean approaches have been most successful for line extensions and initial MAs. Lean post-approval proposals for DS are more likely to be accepted compared to DP proposals.

Case study 1 A stable drug substance was filed with 6 month $\mathrm{ICH} /$ registration data in the initial registration dossier. For both the post-approval (PA) confirmation and annual commitment lots, reduced tests (only appearance and purity), time points (annually) and condition $\left(25^{\circ} \mathrm{C} / 60 \% \mathrm{RH}\right)$ were proposed and accepted in more than 30 countries (Colgan et al., 2014).

Case study 2 An initial NDA was filed in the US for a combination product consisting of a complex modified release (MR) formulation with multiple strengths using common pellets. The initial registration submission was filed with 36 month primary $\mathrm{ICH} /$ registration data at the long-term condition; a 36 month shelf-life was proposed. As the primary batches were manufactured at the commercial scale and site, no shelf-life confirmation was proposed, in-line with ICH Q1A (ICH Q1A(R2), 2003). For the annual lots, testing of only one lot of one strength was proposed, as comparable release and stability data were observed for all strengths and there were no strength-related trends. Further, a reduction in testing (exclude water content and assay), timepoints (annually only) and condition $\left(25{ }^{\circ} \mathrm{C} / 60 \% \mathrm{RH}\right)$ were proposed. After two rounds of queries on the exclusion of water testing, it was agreed to monitor moisture without inclusion on the specification, then to either update via a changes-being-effected in 30 days (CBE30) submission or justify via a general correspondence. For the assay test, a commitment was made to include the test on the protocol, but to file a prior approval supplement (PAS) once further stability data is available.

Case study 3 The initial registration of a stable, well understood DP (immediate release capsule) was filed containing 12 month of primary $\mathrm{ICH} /$ registration data. The proposal for the shelf-life confirmation was to use non-printed capsules for 2 of 3 lots for 2 of 3 strengths (due to low commercial volume). Further reduced testing points and conditions were proposed as follows: $6,12,24$, 36 month at $30{ }^{\circ} \mathrm{C} / 75 \% \mathrm{RH}$ and 6 month at $40{ }^{\circ} \mathrm{C} / 75 \% \mathrm{RH}$. For the annual lots, only appearance, degradation products and dissolution test were proposed, with reduced testing time points and conditions (only $0,12,24$ and 36 months at $\left.30{ }^{\circ} \mathrm{C} / 75 \% \mathrm{RH}\right)$.

The US FDA requested and we agreed to commit to testing assay for the annual lots and time points for the 
Table 3 Examples of Lean Stability Strategies in the Initial and Post-Approval Registration Dossiers

\begin{tabular}{|c|c|c|}
\hline No. & Strategy ${ }^{a}$ & Comments \\
\hline 1 & $\begin{array}{l}\text { Monitor fewer storage conditions, e.g. only at } 30^{\circ} \mathrm{C} / 75 \% \mathrm{RH} \text { to support all filing zones and } \\
\text { global labeling requirements }\end{array}$ & $\begin{array}{l}\text { Justification is included in the submission } \\
\text { (see Colgan ST (2014); Freed AL. (2016) }\end{array}$ \\
\hline 2 & $\begin{array}{l}\text { Monitor reduced time points for DS or DP (immediate release, complex modified release) } \\
\text { post-approval confirmation and/or annual commitment protocols }\end{array}$ & \\
\hline 3 & Employ bracketing or bracketing and matrixing when applicable & \\
\hline 4 & $\begin{array}{l}\text { Monitor only attributes that are stability related quality attributes (SQRAs) or the single shelf } \\
\text { life limiting attribute (SLLA) }\end{array}$ & \\
\hline 5 & $\begin{array}{l}\text { Consider including planned/anticipated post-approval changes (PACs) in the initial Marketing } \\
\text { Application (MA) (e.g. in the stability sections of the dossier itself (potential change) or as a } \\
\text { post-approval change management protocol) as possible }\end{array}$ & \\
\hline 6 & $\begin{array}{l}\text { Using ASAP, SRA, statistics, etc. to assess PACs, thereby reducing number of lots, time } \\
\text { points, etc. }\end{array}$ & \\
\hline 7 & $\begin{array}{l}\text { Exclusion of site specific stability information, even for those markets that require site } \\
\text { specific stability }\end{array}$ & \\
\hline
\end{tabular}

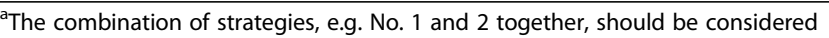

shelf life confirmation program. The Chile Health Authority approved confirmation of shelf life strategies for 1 of 2 packaging types. Submissions have taken place and are pending in numerous countries/regions globally.

Case study 4 An initial registration for a DP line extension of a stable established product (new formulation; tablet) included 12 month of primary $\mathrm{ICH} /$ registration data. A reduction in the testing (appearance, degradation products and disintegration only), timepoints (annual) and conditions $\left(30{ }^{\circ} \mathrm{C} / 75 \% \mathrm{RH}\right.$ only) was proposed for both PA confirmation \& annual commitment protocols. Further, no commercial site stability data was provided in the registration dossiers submitted to markets that required this data. In some cases, the commercial site data was requested, but the lean stability protocol strategy was successful in nearly all cases.

\section{Overcoming regulatory challenges: things to consider and next steps}

Lean stability is here to stay and is securing more converts each year, as can be seen in part by Innovation and Quality (IQ) Consortium activities, as the Analytical Leadership Group approval of the Lean Stability Working Group formation in June 2016. We are making progress in the understanding, application and acceptance of science and risk based approaches, including lean stability. There are several different modeling tools and strategies that can be leveraged. When including lean stability approaches in a dossier, there are several points should be considered to increase the likelihood of success. For example, sponsors should:

- Engage in discussions with the Health Authority when possible (especially in the registration phase);
- Include clear and concise justifications;

- Include pertinent discussion of the "toolkit" (e.g. modeling tools) used;

- Be prepared to provide additional data, justification;

- Be flexible and prepared to compromise;

- Be prepared to be told 'no' more than once;

- Don't be afraid to try again, e.g. once additional data is available (statistics can be a powerful tool!).

Industry and regulators need to collaborate to generate meaningful risk-based stability guidance that meets both the scientific and regulatory requirements. To leverage this momentum, we need to continue to socialize the concept internally (across division) and externally (industry forums globally, white papers), focus on meaningful data and risk-based strategies and move away from a "one size fits all" approach. Further, industry should continue to partner to identify best practices and facilitate ongoing dialog with regulators. Moreover, sponsors need to capitalize on alternative approaches allowed in current country regulatory guidances. Fig. 2 shows several ways in which each of us can contribute to this important effort. Together we can continue this momentum!

\section{Part II}

Analytical quality by design for methods development in the analytical life cycle management as prepared by: Jianmei Kochling, Sanofi

In the 2016 stability workshop, there was a dedicated section for analytical life cycle management and analytical quality by design for stability method development and validation. Topics discussed were as follows: Patrick Faustino, PhD, US FDA, "Analytical Method Strategies for Large Molecules Method Validation to Achieve Stability Model Objectives", Dilip Choudhury, PhD, Pharmaceutical Consultant, "A Lifecycle Strategy for Analytical 


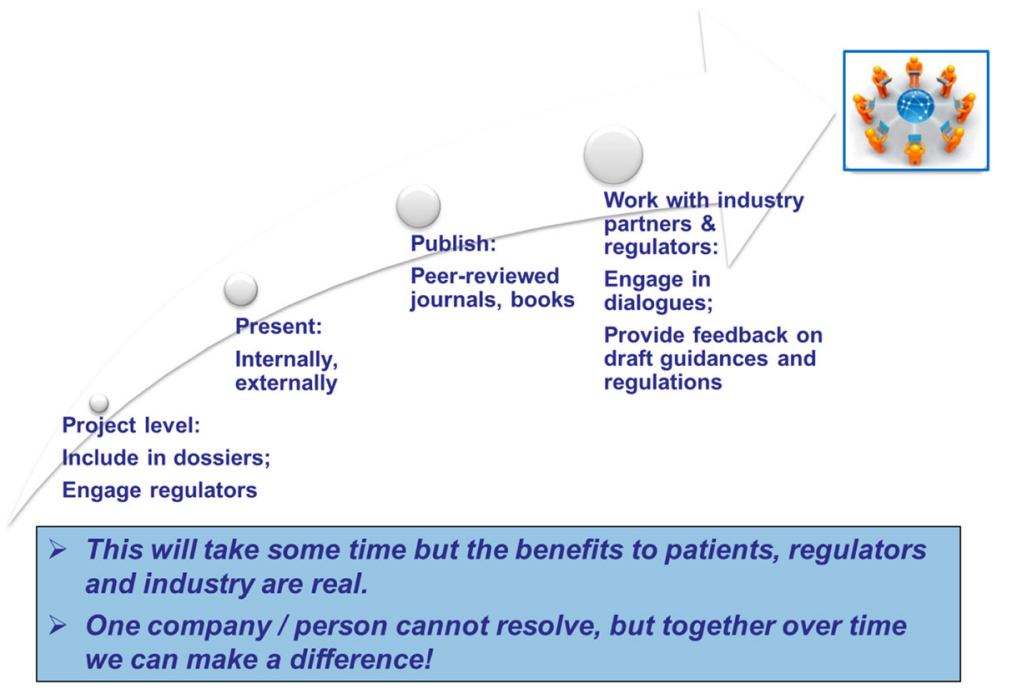

Fig. 2 How Can I Help? (permitted to reprint)

Method Design to Support Stability Modeling", Jianmei Kochling, PhD, Sanofi, "QbD for Method Development and Validation for Large Molecules"; and Saji Thomas, MS, Par Pharmaceutical, an ENDO International Company, "Life Cycle Management of Analytical Methods for Small and Large Molecules-Case Studies". A summary of these discussions is presented below. An excellent example of the application of QbD for method development can be found in the following reference (Kochling et al., 2016).

\section{Analytical quality by design (AQbD) concept}

The quality by design concept has been well adapted by analytical chemists and termed"analytical quality by design" (AQbD). AQbD is a systematic methods development strategy which consists of an overall consideration of scientific and regulatory knowledge, as well as quality control needs. This strategy ensures that method robustness is built in during development.

The AQbD work flow is like the process flow in $\mathrm{QbD}$ for drug development as depicted in Fig. 3. Similar to QbD for drug development, AQbD starts with defining the intended purpose of the method and its analytical target profile (ATP). Each analytical method should have an intended purpose, whether it is to be used to support research, process and formulation development, release and stability testing for clinical or marketed drugs, quantitative, qualitative, or limit tests. The method ATP combines the intended purpose and the ICH Q2R analytical method quality requirements: specificity, precision, accuracy, linearity, range, quantitation limit, and detection limit. Through method scouting experiments, the most suitable analytical technique is chosen, (e.g.,

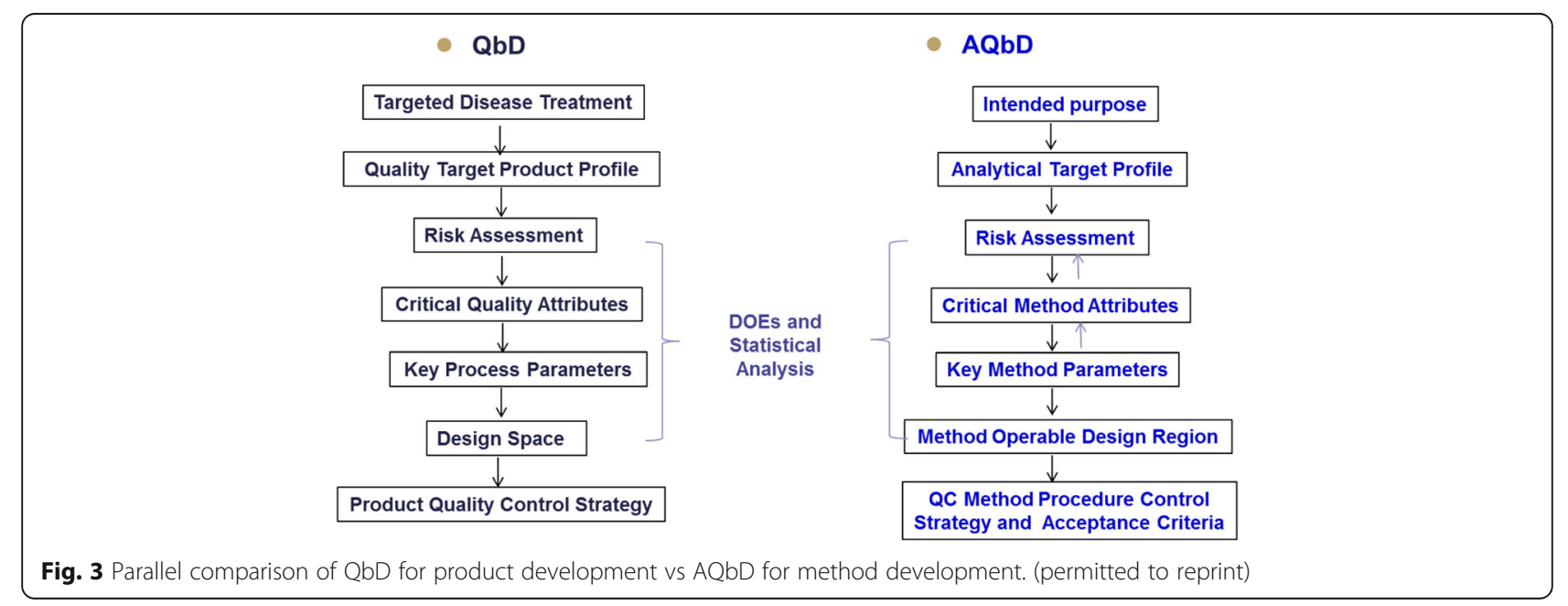


whether the method uses UHPLC-UV or UHPLC-MS or fluorescence labeling), and the method is then developed to meet its intended purpose.

The determination of potential critical method attributes (CMAs) is an analogue of critical quality attributes (CQAs) in drug development. In analytical method development, method attributes are generally defined as the steps the method must have, such as sample preparation, sample introduction, sample analysis, and data analysis. As with process parameters, the method parameters are the detailed steps of a method attribute, such as the extraction time for sample preparation. Determining these key method parameters (KMPs) is analogous to determining the critical quality attributes CQAs as part of a $\mathrm{QbD}$ approach to drug product development. Finally, the method quality control strategy is established from the understanding of the criticality of each method parameter for each method attribute. Controls can then be set as procedures or acceptance criteria in the analytical method - analogous to the concept of control strategy (specification) for the drug product development. The AQbD flow diagram can be found in Fig. 4.

\section{Analytical Life Cycle Management}

The lifecycle concept described in ICH Q8 (Pharmaceutical Development) (ICH, 2017) has also been adapted to analytical methods with the parallel phrase: "Analytical Life Cycle Management (ALCM)". Analytical methods have life cycles, going from clinical to commercial phase as processes and formulations are changed. When the analytical methods are validated and implemented for routine use, unforeseen issues will occur during their use. The methods should be assessed for the frequency of out-of-specification and out-of-trend data, data accuracy and variability, failure rate, easiness of method execution, and cost of operation. These details guide the effort for the next generation of method improvement. In addition, method requirements may change, as product specifications become tighter. Thus, the analytical method need to be reassessed and changed accordingly to remain suitable for use throughout the drug development process.

Application of ALCM ensures that analytical methods evolve as more product and process knowledge and understanding of the analytical method's variability are gained through years of operation. The evolved methods will provide better reliability with reduced variability, generating data that accurately reflects the product quality attributes. Although method development strategy as depicted in Fig. 4 applies to any dosage form of drugs, small molecules and large molecules, solid vs parenteral dosage forms, each of these have their own scientific knowledge and regulatory requirements that also must be met.

\section{Advantages of $A Q b D$ applications for method development for large molecules}

For large protein molecules, a platform AQbD approach is a very effective way to improve method development efficiency, quality, and reduce operation cost. Large molecules, although very complicated in structure, can often share the same analytical procedures, conditions, and equipment across a range of applications. They can offer a technology-based method application for multiple products, the same type of method for different products or different methods for one product with common procedures, until the diverging point; whereby, product specific procedures must be applied. For example, the platform

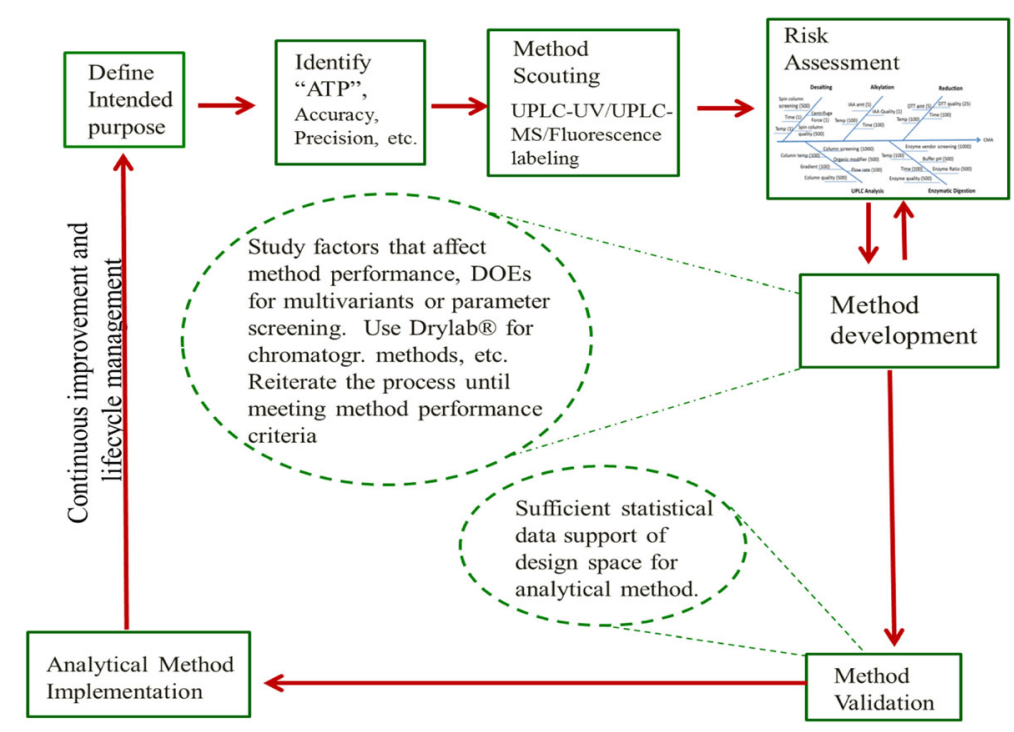

Fig. 4 A flow diagram illustrating the process of analytical quality by design (AQbD) (permitted to reprint) 
AQbD approach applied to different proteins with the same sample preparation steps for reduction, alkylation, desalting, and digestions; different UHPLC methods with the same column and buffers; the same CE-SDS method application to different proteins, the same glycan sample preparation and analysis methods for different proteins, etc. The AQbD approach requires the development team to think broadly in considering as many methods as possible during the steps of "defining intended purpose, ATP, and method scouting". Once the final method technology is determined, one should screen across different batches of product before finalizing the method.

In $\mathrm{AQbD}$, the use of design of experiments or modeling software is emphasized, this offers unbiased experimental approaches and data output which guide correct decision making. With the robustness of the methods being builtin during method development, error rate will be reduced and accurate data will be generated. Which builds a solid database for product life cycle management.

\section{$A Q b D$ section summary}

There is a significant advantage applying $\mathrm{QbD}$ to analytical method development, regardless of molecule size, small molecules or large molecules, the work flow is the same as shown in Fig. 4. An AQbD approach enhances efficiency and reducse cost. Plus, the method development knowledge gained for one molecule can be used for others since the technology can be similar. For small molecules, shared technology, such as HPLC can be used; and, for large molecules, similar techniques may also be used. From an ALCM stand point, going from clinical to commercial phase, methods will evolve and the knowledge gained can be used for future programs. This further emphasizes the need for knowledge management during analytical life cycle management.

\section{Workshop conclusion}

Overall the meeting was a success. The meeting offered an opportunity to discuss the latest developments and successes of predictive stability approaches with our colleagues in industry, FDA and USP and listen to their feedback. There is an openness to the approach, especially as evidenced by the increasing global regulatory acceptance of Lean Stability Strategies. Much progress has been made since the early introduction of ASAP and the Lean Stability concept; and, more is needed to achieve the utopian vision of no additional stability testing after batch release. This progress may be accelerated using programs that pull from industry and internet data bases, such as Zenith for predicting degradation products and ReadAcross for predicting a molecule's safety assessment liability.

\section{Acknowledgements}

The authors gratefully acknowledge the subject matter experts that participated in this workshop and AAPS for sponsoring the workshop. The authors would also like to acknowledge those that contributed to the Lean Stability initiative: Beth Kendsersky, Connie Langer, Greg Sluggett, Ken Waterman, Rob Timpano, Elise Clement, Kevin Ryan, Keith Masse, Dorys Diaz, Doug Farrand.

\section{Authors' contributions}

$\mathrm{AF}$ and SC wrote the section on Lean Stability, JK wrote the section on Analytical QbD, and MA prepared all other sections. All authors read and approved the final manuscript.

\section{Competing interests}

All authors are employees of the companies and receive payments from their companies.

\section{Publisher's Note}

Springer Nature remains neutral with regard to jurisdictional claims in published maps and institutional affiliations.

\section{Author details}

${ }^{1}$ Pfizer WRD, Groton, CT, USA. ${ }^{2}$ Sanofi, Framingham, MA 01701, USA.

${ }^{3}$ Orexigen Therapeutics, Inc, La Jolla, CA 92037, USA.

Received: 15 March 2017 Accepted: 14 August 2017

Published online: 06 September 2017

\section{References}

AAPS Joint Face-to-Face meeting summary, 6-April, part1, http://www.aaps.org/ uploadedFiles/Functional/AAPS_Focus_Group_Discussion_Board/Stability_ Focus_Group/2016\%20Final\%20F2F\%20Joint\%20Meeting\%20Highlights_ final_\%20part\%201.pdf. Accessed 20 Aug 2017

AAPS Joint Face-to-Face meeting summary, 6-April, Part II, http://www.aaps.org/ uploadedFiles/Functional/AAPS_Focus_Group_Discussion_Board/Stability_ Focus_Group/2016\%20Final\%20F2F\%20Joint\%20Meeting\%20Highlights_ final_part\%202.pdf. Accessed 20 Aug 2017

AAPS Annual Meeting Sunrise Session (2016) Accelerating Pharmaceutical Development through Predictive Stability Approaches, AAPS Annual Meeting, 16 November, 2016

AAPS Workshop, Accelerating Pharmaceutical Development through Predictive Stability Approaches, 4-5 April, 2016

Alasandro MS, Little TA (2014) Multifactor Non-linear Modeling for Accelerated Stability Analysis and Prediction. Pharm Technol 38(7):46-49

Alasandro, MS, Little, TA (2015) Process and Method Variability Modeling to Achieve QbD Targets, AAPS Pharm Sci Tech, 2016 Apr;17(2): 523-527. doi: https://doi.org/10.1208/s12249-015-0380-3 Accessed 29 May 2017

Alasandro MS, Little TA, Fleitman J (2013) Method Validation by Design to Support Formulation Development. Pharm Technol 37(4):84-92

CHMP/QWP/185401/2004 (2006) Guideline on the Requirements to the Chemical and Pharmaceutical Quality Documentation Concerning Investigational Medicinal Products in Clinical Trials. http://www.ema.europa.eu/docs/en_GB/ document_library/Scientific_guideline/2016/04/WC500204674.pdf. Accessed 20 Aug $20 \overline{17}$

Colgan S, Hofer J, Timpano R, Vukovinsky K, Waterman K, Norris K (2015) Lean Stability. AAPS News Mag 2015:14-18

Colgan ST (2016) Integrating Stability Modeling Tools into a Lean Stability Design - Case Studies. 4-5 April, 2016

Colgan ST, Timpano J, Roberts M, Weaver R, Ryan K, Fields KW et al (2014) Opportunities for Lean Stability Strategies. J Pharm Innov 9(4):259-271

FDA Final Report Pharmaceutical cGMPs for the 21st Century - A Risk-Based Approach.; September 2004. https://www.fda.gov/downloads/drugs/ developmentapprovalprocess/manufacturing/ questionsandanswersoncurrentgoodmanufacturing practicescgmpfordrugs/ ucm176374.pdf. Accessed 20 Aug 2017

FDA Guidance for Industry: Content and Format of Investigational New Drug Applications (INDs) for Phase 1 Studies of Drugs, Including WellCharacterized, Therapeutic, Biotechnology-Derived Products.; November 1995. https://www.fda.gov/downloads/drugs/ guidancecomplianceregulatoryinformation/guidances/ucm071597.pdf. Accessed 20 Aug 2017 
FDA Guidance for Industry: INDs for Phase 2 and Phase 3 Studies, CMC Information.; May 2003 https://www.fda.gov/downloads/Drugs/Guidances/ ucm070567.pdf. Accessed 20 Aug 2017

Freed AL. (2016) Lean Stability: Global Regulatory Reception - Successes and Challenges of Recent Case Studies. 4-5 April, 2016

Freed AL, Clement E, Timpano R (2014) Regulatory Responses to the Use of Various Lean Stability Strategies in Early Drug Development. Regul Rapporteur 11(7/8):5-8

Health Canada Quality (Chemistry and Manufacturing) Guidance: Clinical Trial Applications (CTAs) for Pharmaceuticals; 2013. http://www.hc-sc.gc.ca/dhpmps/alt_formats/hpfb-dgpsa/pdf/prodpharma/qual_cta_dec-eng.pdf. Accessed 20 Aug 2017

Huynh-Ba, et al (2017) Meeting Report, Analytical Approaches to Ensure Product Quality - AAPS Joint Face-to-Face Meeting of the Stability, the Pharmaceutical Impurities, and the CMC Statistics Focus Group, April $6^{\text {th }}$, 2016 in Gathersburg, MD

ICH Q8(R2) (Pharmaceutical Development) http:/www.ich.org/fileadmin/Public Web_Site/ICH_Products/Guidelines/Quality/Q8_R1/Step4/Q8_R2_Guideline. pdf. Accessed 20 Aug 2017

ICH Q1A(R2) Stability Testing of New Drug Substances and Products.; February 2003. http://www.ich.org/fileadmin/Public_Web_Site/ICH_Products/ Guidelines/Quality/Q2_R1/Step4/Q2_R1__Guideline.pdf. Accessed 20 Aug 2017

ICH Q1D Bracketing and Matrixing Designs for Stability Testing of New Drug Substances and Products.; February 2002. http://www.ich.org/fileadmin/ Public_Web_Site/ICH_Products/Guidelines/Quality/Q1D/Step4/Q1D_ Guideline.pdf. Accessed 20 Aug 2017

Kochling J, Wu W, Hua Y, Guan Q, Castaneda-Merced J (2016) A platform analytical quality by design (AQbD) approach for multiple UHPLC-UV and UHPLC-MS methods development for protein analysis. J Pharm Biomed Ana 125:130-139

Langer C, Carella A, Colgan S. Lean (2016) Post Approval Stability Strategies as a Means to Efficiently Safeguard Quality, AAPS Annual Meeting, November 14, 2016

Lavrich, D (2016) Rapid Development of Robust Stability Models Using SemiEmpirical Design Space, AAPS Webinar, 24 March 24, 2016

Li H, Nadig D, Kuzmission A and Riley CM. Prediction of the changes in drug dissolution from an immediate-release tablet containing two active pharmaceutical ingredients using an accelerated stability assessment program (ASAPprime ${ }^{\circledast}$ ), AAPS Open (2016) 2:7 doi: https://doi.org/10.1186/ s41120-016-0010-5

Timpano R, Freed AL, Clement E, Masse K, Ryan K. (2017) ASAP Regulatory Strategy and Acceptance and Feedback in Accelerated Predictive Stability: Fundamentals and Pharmaceutical Industry Practices, first edition, Elsevier, 2017

Waterman K, Carella A, Gumkowski M, Lukulay P, MacDonald B, Roy M et al (2007) Improved Protocol and Data Analysis for Accelerated Shelf-Life Estimation of Solid Dosage Forms. Pharm Res 24(4):780-790

WHO guideline Annex 2 Stability testing of active pharmaceutical ingredients and finished pharmaceutical products.; 2009. http://apps.who.int/ medicinedocs/documents/s19133en/s19133en.pdf. Accessed 20 Aug 2017

Wu Y, Freed AL, Lavrich D, Raghavachari R, Huyhn-Ba K, Shah K, Alasandro MS (2016) Bringing Drug Product Marketing Applications to Meet Current Regulatory Standards: Trials and Tribulations. AAPS Pharm Sci Tech 16(4):986-991. doi:10.1208/s12249-015-0334-9 Accessed 29 May 2017

Zeneth by Lhasa Limited. [Online]. Available from: http://www.lhasalimited.org/ products/zeneth.htm. Accessed 20 Aug 2017

\section{Submit your manuscript to a SpringerOpen ${ }^{\circ}$ journal and benefit from:}

- Convenient online submission

- Rigorous peer review

- Open access: articles freely available online

- High visibility within the field

- Retaining the copyright to your article 